\title{
Forest vintages and carbon sequestration
}

\author{
Clara Costa-Duarte, Maria A. Cunha-e-Sá, Renato Rosa \\ Universidade Nova de Lisboa, Faculdade de Economia \\ Campus de Campolide, P-1099-032 Lisboa, Portugal
}

May 12, 2006

\begin{abstract}
In the current paper we examine the role of forest carbon sequestration benefits in optimal forest management. When carbon benefits are considered not only the forested area is relevant, but also the flow of carbon between land and the atmosphere through the carbon cycle. To account for all these impacts a multi-vintage forest setting is used, following Salo and Tahvonen (2004). The model is extended to three different carbon accounting methods to measure the benefits form carbon sequestration: carbon flow regime, tonne-year crediting and average storage. In the case of the carbon flow regime, the impact on the optimal management and allocation of land will depend upon the amount of carbon released when the forest is harvested. Under the other two accounting systems optimal steady state forest area will be increased, and in cases where optimal management imply cyclical harvesting, considering carbon benefits will always increase cycles dimension.
\end{abstract}




\section{Introduction}

Given the rising concern with $\mathrm{CO} 2$ levels, and the recognition in the Kyoto Protocol of the important role that can be played by forests in the global carbon cycle to limit the impact of GHGs emissions, the consideration of carbon sequestration benefits is in the center of recent developments in forestry literature. Thus, in order to allocate credits to forest owners, carbon sequestration benefits' accountability has to be thoroughly addressed.

In the context of the related literature, we should mention, among others, Van Kooten, Binkley and Delcourt [5], who modeled a scheme to allocate carbon credits, under which the carbon credit cash flows are a function of the annual change in the forest carbon stock (carbon flow regime), Spring, Kennedy, and Nally [8] that study the effect of carbon sequestration, fire frequency and water scarcity in tree harvest decision, and Cunha-e-Sá and Rosa [6] where different accounting methods of carbon sequestration benefits in the model of the private forester are examined with constant and rising carbon prices. Also, while Velt and Plantinga [7] explore the effect of rising carbon prices on the optimal portfolio of greenhouse-gas mitigation strategies based on the carbon flow accounting regime, Sohngen and Mendelsohn [4] develop an optimal control model of carbon sequestration and energy abatement to explore the potential role of forests in GHG mitigation using a simplified version of the tonne-year crediting accounting regime.

When carbon benefits are considered not only the forested area is relevant, but also the flow of carbon between land and the atmosphere through the carbon cycle, namely, the amount of carbon released when the forest is harvested. To account for all these impacts the usual analytical framework of one stand forest, used in most of the recent literature, is insufficient. In a one stand forest neither the decision on the optimal allocation between alternative uses is taken into 
account, nor the fact that forested areas contribute in a permanent way to the carbon cycle. Therefore, a multi-vintage forest setting with possible conversion to alternative land uses should be considered instead.

The model used in this paper follows closely the multiple vintage forest model developed in Salo and Tahvonen [1], [2] and [3], extending their results to the case where optimal use of land also considers the benefits from carbon sequestration. The line of work developed by these authors has been able to provide a full proof on the log-run optimality of the normal forest steady-state, and as referred in Salo and Tahvonen [3] "...could provide a generalized and computational efficient modeling structure...for studies on carbon sequestration, deforestation or timber supply". ${ }^{1}$

The present paper focus on the impact of different carbon accounting methods on optimal land allocation and optimal forest management. The social planner's decision problem on forest harvesting and land allocation is studied in those cases extending the proofs on the existence of optimal stationary steady-states to this more general context. Besides, we characterize the optimal solutions, and compare the results obtained with those without carbon sequestration benefits. Finally, numerical examples are presented and discussed to illustrate the results obtained.

In general, we conclude that the main results in Salo and Tahvonen [3] still apply. In fact, when we consider benefits from carbon sequestration, in the case where all land is forested land, optimal forest management can still lead to optimal cyclical harvesting and when it is optimal to allocate part of the forest land to alternative land use, the remaining equilibrium is the normal forest steady state. In addition, the optimal allocation area to forest will increase, as the net value from accounting carbon sequestration benefits is positive. Finally, the claim that the accounting method of carbon benefits is relevant for the

\footnotetext{
${ }^{1}$ See Salo and Tahvonen [3], pages 526-527.
} 
optimal forest management is reinforced, as it is clearly shown that different accounting methods determine different impacts on both optimal cycles and optimal steady states.

The remainder of the paper is organized as follows. Section 2 presents the different accounting methods of carbon sequestration benefits, Section 3 extends the theoretical multiple vintage model to account for carbon sequestration benefits. Section 4 develops the model for the three carbon accounting methods considered: the carbon flow regime, the ton-year crediting and the average storage method. Section 5 concludes the paper. Technical details are presented in the Appendices.

\section{Accounting methods}

By sequestering and storing GHG's from the atmosphere, forests can generate carbon offsets, which may be used to compensate for GHG emissions. However, for this compensation to occur, the net effect of sequestration has to be comparable to that of avoided emissions. This issue raises two important questions: first, how to compare forest carbon sequestration with avoided emissions, and second, how to incorporate the services provided by this activity when modeling forest management, which depends upon the choice of the carbon accounting method.

The IPCC Special Report on Land Use, Land-Use Change and Forestry [9] considers different accounting methods to apply to forest or land use change investment projects, namely, the stock change method, the average stock method and the tonne yearly crediting. In the economic forestry literature, similar accounting methods have also been considered: the carbon flow regime, the

lump-sum regime, and the carbon stock regime, among others, as referred in Locatelli and Pedroni [10]. 
According to the carbon flow regime, as developed in Van Kooten, Binkley and G. Delcourt [5], for the implementation of a carbon credit cash flow, social benefits are a function of the annual change in the forest carbon stock. A net increase in the forest carbon stock over a year means that carbon has been removed from the atmosphere. Similarly, a fall in the forest carbon stock suggests that carbon has been released into the atmosphere. To an increase in the forest standing biomass corresponds an increase in the carbon stock, and harvesting a forest decreases the carbon stock. However, the amount of carbon released when the forest is harvested depends upon the use given to the timber harvested. Different uses will have different impacts on the amount of carbon released after harvest, as some uses are able to provide long term carbon storage in structures like furniture or houses.

An alternative approach is the tonne-year crediting regime. The ton-year method consists of crediting a forestry project with a fraction of its total yearly GHG benefit, based on what is called an equivalence factor $\left(E_{f}\right)$. This fraction is determined by the stock of carbon stored each year, which is then converted, using $\left(E_{f}\right)$ to its equivalent amount of preventing effect. Notice that this method does not require redemption of carbon credits upon harvest. Within this approach, two different methods have been proposed by Moura-Costa and Wilson [11], and by Fearnside, Lashof and Moura-Costa [12]. In both, the calculations are based on the residence time and decay pattern of atmospheric $\mathrm{CO} 2$, its Absolute Global Warming Potential (AGWP), taking explicitly into account the decay pattern of GHGs in the atmosphere.

Moura Costa and Wilson [11] aim to determine the storing time of carbon sequestered in biomass for which the carbon stored is equivalent to an amount of avoided emissions (equivalence time). It was found that keeping a megagram $(\mathrm{Mg})$ of $\mathrm{CO} 2$ out of the atmosphere for a full 100 years represents $55 \mathrm{Mg}$-year (or ton-year) equivalents, rather than the $100 \mathrm{Mg}$-years that would be earned if 
the CO2 entering the atmosphere had no movement to the ocean or other sinks. The number obtained, in this case, 55 , is denoted by the equivalent time, $T_{e}$. In addition, assuming a linear relationship between the residence of $\mathrm{CO} 2$ in the atmosphere and its radiative forcing effect, the effect of storing 1 tonne of $\mathrm{CO} 2$ in forest biomass for 1 year was derived. In particular, it was found that storing one ton of carbon for one year is equivalent to preventing the effect of 0.0182 tones of $\mathrm{CO} 2$ emissions, which is denoted by equivalence factor $\left(E_{f}=1 / T_{e}\right)$.

Also based on a Absolute Global Warming Potential (AGWP) function, Fearnside et al. [12] estimate the incremental credit that can be awarded for each year that carbon stocks remain sequestered. For this purpose these authors assume as the benchmark "keeping a $\mathrm{Mg}$ of $\mathrm{C}$ out of the atmosphere for a full 100 years". If the stock remains intact for 100 years, the cumulative awarding of ton-year credits would equal the credits from a "permanent" emission reduction of the same magnitude. If the stock is released at any time prior to the 100-year time horizon, only the corresponding partial credit amount would be awarded.

Finally, the average carbon storage method consists of averaging the amount of carbon stored in a site over the long run, assuming an average cycle rotation period. By simplifying the process of credit allocation between the forest owners and the regulatory agencies, this method is more efficient than both the carbon flow and the tonne-year crediting ones. These three accounting methods will be formally considered in the remainder of the paper.

\section{The Model}

The model used in this paper follows closely the multiple vintage forest model developed in Salo and Tahvonen [3], which can be summarized as follows. The

model assumes multi vintages forest land, where $s=1, \ldots, n$ represents the age of trees, $x_{s, t}$ the area of forest land allocated to the age class $s$ in period $t, f_{s}$ 
the biomass content in timber per unit of land with trees of age class $s$, and $0 \leq f_{1} \leq \ldots \leq f_{n}$. Land allocation must satisfy

$$
0 \leq y_{t}=1-\sum_{s=1}^{n} x_{s, t}
$$

that is, total land area equals 1 , and $y_{t}$ is the area of land allocated to an alternative use (agriculture or urban use).

Social utility of land use in period $t$ can be derived from timber consumption $c_{t}$ and carbon sequestration $S_{t}$, or from social utility of the alternative use of land $W\left(y_{t}\right)$.

Let us denote by $U\left(c_{t}\right)=\int D(c) d c$ the social utility from timber consumption, where $D($.$) is the inverse demand for timber, and assume U($.$) is a con-$ tinuous, twice differentiable, increasing and strictly concave function. Also, $W\left(y_{t}\right)=\int Q(y) d y$, where $W($.$) is a continuous, twice differentiable, increasing$ and concave function. Finally, $S_{t}$ depends on the way the benefits from carbon sequestration are accounted for, as shown in Section 4.

Thus, the problem of optimal forest harvesting with carbon sequestration benefits and allocation of land is obtained by maximizing the present value of social utility from the use of land as follows:

$$
v\left(x_{1,0}, \ldots . x_{n, 0}\right)=\underset{\left\{x_{s, t+1}, s=1, \ldots n, t=0, \ldots\right\}}{\operatorname{Max}} \sum_{t=0}^{\infty} b^{t}\left[U\left(c_{t}\right)+S_{t}+W\left(y_{t}\right)\right]
$$

subject to

$$
\begin{gathered}
c_{t}=\sum_{s=1}^{n-1} f_{s}\left(x_{s, t}-x_{s+1, t+1}\right)+f_{n} x_{n, t} \\
y_{t}=1-\sum_{s=1}^{n} x_{s, t} \\
x_{s+1, t+1} \leq x_{s, t}, s=1, \ldots \ldots n-1
\end{gathered}
$$




$$
\begin{gathered}
\sum_{s=1}^{n} x_{s, t+1} \leq 1 \\
x_{s, t} \geq 0, s=1, \ldots, n
\end{gathered}
$$

for all $t=0,1 \ldots$, where $S_{t}$ is given by (11), (30), or (44), respectively, depending on the particular carbon benefits accounting method used. Finally, the initial land distribution satisfies

$$
x_{s, 0} \geq 0, s=1, \ldots, n, \sum_{s=1}^{n} x_{s, 0} \leq 1
$$

Therefore, given the discount factor $b$, the problem is to choose the next period state, that is, the land allocation between different vintages and competing uses of land for all $t=1, \ldots$

The necessary conditions for optimal solutions can be obtained from the following Lagrangian problem. For (2-8) it can be stated as :

$L=\sum_{t=0}^{\infty} b^{t}\left[U\left(c_{t}\right)+S_{t}+W\left(y_{t}\right)\right]+\lambda_{t}\left(1-\sum_{s=1}^{n} x_{s, t+1}\right)+\sum_{s=1}^{n-1}\left[p_{s, t}\left(x_{s, t}-x_{s+1, t+1}\right)\right]$

where $p_{s, t}$ and $\lambda_{t}$ are the Lagrangian multipliers. While $p_{s, t}$ can be interpreted as the value of marginal changes in forest land area of vintage $s$ at the beginning of period $t+1, \lambda_{t}$ represents the value of marginal changes in land allocation between forest and alternative uses.

Salo and Tahvonen [3] provide a full proof on the log-run optimality of the normal forest steady-state for the above problem, when $S_{t}=0$.

In this paper a similar definition for normal forest steady state is assumed. Denote the Optimal rotation period by $m$, that satisfies $1 \leq m \leq n$ and

$$
b^{m} f_{m} /\left(1-b^{m}\right) \geq b^{s} f_{s} /\left(1-b^{s}\right), s=1, \ldots, n .
$$


Assume that $m$ is unique. ${ }^{2}$

A forest is called an Optimal Steady-State Forest (OSSF) if the age-class structure $x=\left(x_{1}, \ldots, x_{n}\right)$ has the property $x \in S, x_{s}=0$ for $s=m+1, \ldots, n$ and if harvesting only trees of age $m$ is the optimal solution for the above problem when $x_{0}=x$. An OSSF is an interior OSSF if $x_{s}>0$ for $s=1, \ldots, m$.

An OSSF with the normal forest structure is $x=(1 / m, \ldots, 1 / m, 0, \ldots, 0)$, and in each period it yields a constant consumption level of $f_{m} / m$. An OSSF with consumption that is periodic with period length equal to $m$ can be expressed as $x=\left(1 / m+\phi_{1}, \ldots, 1 / m+\phi_{m}, 0, \ldots 0\right) \in S$. Define $\phi^{k}$ as the largest number $\phi$ that satisfies $x=\left(1 / m+\phi_{1}, \ldots, 1 / m+\phi_{m}, 0, \ldots 0\right) \in K$ for all $\left|\phi_{s}\right|<\phi$, $s=1, \ldots, m, \sum_{s=1}^{m} \phi_{s}=0$.

For the case $S_{t}=0$, Salo and Tahvonen [3] show that, if all land is allocated to forestry, optimal forest management can lead to optimal cyclical harvesting because smoothening an age class structure that deviates from the normal forest is not optimal. On the contrary, if it is optimal to allocate part of the land to alternative land use then optimal stationary cycles cannot exist. ${ }^{3}$ In this paper, these results are extended to the case where forest management considers carbon sequestration benefits, i.e. $S_{t} \neq 0$.

\section{Introducing Carbon Sequestration Benefits}

In this section, we introduce carbon sequestration benefits in the forest-vintage model presented in Section 3. As mentioned before, we consider three different carbon accounting methods to which correspond a different specification for net carbon benefits: the carbon flow regime, the tonne-year crediting and the

\footnotetext{
${ }^{2}$ In Salo and Tahvonen [3] no harvesting or plantation costs are considered nor any type of forest externalities. Under these conditions $m$ as defined in (10) is also equivalent to the Faustmann rotation period in a one stand model and the authors named the normal stedystate forest as Optimal Faustman Forest. This is no longuer the case when we introduce carbon benefits. Therefore, the normal steady state forest is here renamed as Optimal Steady State Forest.

${ }^{3}$ See Salo and Tahvonen [3], Proposition 1 and Corollary 1, pages 518-520.
} 
average carbon storage. For these three cases, the age-class and land allocation forestry decision problem of the social planner is presented and the necessary and sufficient optimality conditions are derived. By endogeneizing the amount of land allocated to forest use, it is possible to show how the equilibrium cycles are affected by that possibility, and compare the results with the case without carbon sequestration benefits.

When formalizing net carbon benefits, we assume that the social value of one unit of carbon removed from the atmosphere is constant and equal to $P_{c} .{ }^{4}$ Also, we consider that the amount of carbon per cubic feet of timber biomass growing in forest land is constant and equal to $\beta$.

\subsection{Carbon flow regime}

The carbon flow regime considers that to an increase in forest standing biomass corresponds an increase in the carbon stock, and that harvest reduces the carbon stock. Notice that once carbon has been sequestered, no further carbon benefits will be obtained. Thus, in this case, what is relevant when modeling carbon sequestration benefits in a standing forest is the change in the per period carbon uptake. Finally, to take into account different uses of timber we introduce a parameter $\theta$ which measures the fraction of timber that is harvested but goes into long-term storage in structures and landfills.

Under these assumptions, the current net benefits from carbon sequestration at any period $t, S_{t}$, can be represented as follows:

$$
S_{t}=P_{c} \beta f_{1} x_{1, t}+\sum_{s=2}^{n} P_{c} \beta\left(f_{s}-\frac{f_{s-1}}{b}\right) x_{s, t}-P_{c} \beta(1-\theta) c_{t}
$$

where the first two terms represent the value of the carbon stock increase in forest standing biomass, in period $t$, for all the area of forest land, and the last

\footnotetext{
${ }^{4} P_{c}$ is the present value, for all time, of removing one unit of carbon from the atmosphere today. It is determined as the discounted value of the annual contribution to damage caused by one unit of carbon added over the expected number of years that the unit of carbon is present in the atmosphere.
} 
term represents the value of the decrease in the carbon stock due to timber harvesting.

\subsubsection{The necessary conditions for optimal solutions}

The necessary conditions for optimal solutions of the problem (2-8) and $S_{t}$ given by (11), which can be derived from the Karush-Kuhn-Tucker conditions for all $t=0, \ldots$, are as follows:

$$
\begin{aligned}
& b^{t} \frac{\partial L}{\partial x_{1, t+1}}=b f_{1} U^{\prime}\left(c_{t+1}\right)+b f_{1} p_{c} \beta-b f_{1} p_{c} \beta(1-\theta)- \\
& -b W^{\prime}\left(y_{t+1}\right)-\lambda_{t}+b p_{1, t+1} \leq 0 \\
& b^{t} \frac{\partial L}{\partial x_{s+1, t+1}}=-f_{s} U^{\prime}\left(c_{t}\right)+b f_{s+1} U^{\prime}\left(c_{t+1}\right)+b\left(f_{s+1}-\frac{f_{s}}{b}\right) p_{c} \beta+f_{s} p_{c} \beta(1-\theta)- \\
& -b f_{s+1} p_{c} \beta(1-\theta)-b W^{\prime}\left(y_{t+1}\right)-\lambda_{t}+b p_{s+1, t+1}-p_{s, t} \leq 0 \\
& -b f_{n} p_{c} \beta(1-\theta)-b W^{\prime}\left(y_{t+1}\right)-\lambda_{t}-p_{n-1, t} \leq 0 \\
& x_{s, t+1} \geq 0, x_{s, t+1} \frac{\partial L}{\partial x_{s, t+1}}=0, s=1, \ldots, n \\
& p_{s, t} \geq 0, p_{s, t}\left(x_{s, t}-x_{s+1, t+1}\right)=0, s=1, \ldots, n-1
\end{aligned}
$$




$$
\lambda_{t} \geq 0, \lambda_{t}\left(1-\sum_{s=1}^{n} x_{s, t+1}\right)=0
$$

The existence of optimal solutions for bounded utility and $b<1$ follows from Theorem 4.6 in Stokey and Lucas (p. 79).

\subsubsection{On the existence of stationary cycles}

As in Salo and Tahvonen [3], we first study the existence of optimal stationary cycles in a regime where the oldest age class is clear-cut and immediately regenerated at the end of each period and no land is used outside forestry.

Proposition 1 Given $g \equiv \frac{\left[U^{\prime}\left(f_{m} / m\right)+\beta p_{c} \theta\right] b^{m} f_{m}}{1-b^{m}}-\frac{b}{1-b} W^{\prime}(0)>0, m \geq 2$, and $b<1$, there exists a set of interior Optimal Faustmann Forests with $\phi^{k}>0$.

Proof. Following Salo and Tahvonen [3], and using (12) to eliminate $\lambda_{t}$ from (13) and (14), $s=1, \ldots, m-1$, we obtain a system of $m \times(m-1)$ equations:

$b\left(p_{s+1, t+1+k}-p_{1, t+1+k}\right)-p_{s, t+k}=-b\left[U^{\prime}\left(c_{t+1}\right)+\beta p_{c} \theta\right]\left(f_{s+1}-f_{1}\right)+\left[U^{\prime}\left(c_{t}\right)+\beta p_{c} \theta\right] f_{s}$

$$
-b p_{1, t+1+k}-p_{m-1, t+k}=-b\left[U^{\prime}\left(c_{t+1}\right)+\beta p_{c} \theta\right]\left(f_{m}-f_{1}\right)+\left[U^{\prime}\left(c_{t}\right)+\beta p_{c} \theta\right] f_{m-1}
$$

where $s=1, \ldots m-2, k=0, \ldots, m-1$. This system is linear in the Lagragian multipliers $p_{s, t+k}, s=1, \ldots m-1, k=0, \ldots m-1$. Solving for any multiplier yields

$$
p_{s, t}=\frac{b^{m} f_{m}}{1-b^{m}}\left[b^{-s}\left(U^{\prime}\left(c_{t+m-s}\right)+\beta p_{c} \theta\right)-\left(U^{\prime}\left(c_{t}\right)+\beta p_{c} \theta\right)\right]-f_{s}\left(U^{\prime}\left(c_{t}\right)+\beta p_{c} \theta\right)
$$

for $s=1, \ldots, m-1, t=0, \ldots$, as can be verified by direct substitution into the two equations above.

Following Salo and Tahvonen [3], condition (16) requires, for the indefinitely repeated cycle, that $p_{s, t+k} \geq 0$ for $s=1, \ldots m-1, k=0, \ldots, m-1$. Thus, the 
fact that $x \in K$ implies by (20) that

$$
\frac{U^{\prime}\left(c_{t+k}\right)+\beta p_{c} \theta}{U^{\prime}\left(c_{t+k+m-j}\right)+\beta p_{c} \theta} \leq \frac{b^{m-j} f_{m}}{f_{j}+b^{m}\left(f_{m}-f_{j}\right)}
$$

for $k=0, \ldots, m-1, j=1, \ldots m-1$. Using (3) and the definition of Faustmann harvesting, we can write $c_{t+k}=f_{m} x_{s}$ and $c_{t+k+m-j}=f_{m} x_{s-m+j}$ where $s-$ $m+j$ is understood as $s-j$, if $s-m+j \leq 0$. Equation (21) takes the form

$$
\frac{U^{\prime}\left(f_{m} x_{s}\right)+\beta p_{c} \theta}{U^{\prime}\left(f_{m} x_{s-m+j}\right)+\beta p_{c} \theta} \leq \eta_{j} \equiv \frac{b^{m-j} f_{m}}{f_{j}+b^{m}\left(f_{m}-f_{j}\right)}
$$

or, alternatively,

$$
\frac{U^{\prime}\left(f_{m} x_{s}\right)}{U^{\prime}\left(f_{m} x_{s-m+j}\right)} \leq \eta_{j}\left[1-\frac{\beta p_{c} \theta}{U^{\prime}\left(f_{m} x_{s-m+j}\right)}\left(\frac{1}{\eta_{j}}-1\right)\right]
$$

for $s=1, \ldots m, j=1, \ldots, m-1$. Since $\eta_{j}>1, j=1, \ldots, m-1$, is equivalent to $(10)$, and the term in brackets is also positive and greater than 1 , then by the strict concavity of $U$, there must exist a $\phi>0$, such that (22) is satisfied if $x_{s}=1 / m+\phi_{s}, s=1, \ldots, m$, for all $\left|\phi_{s}\right|<\phi, \sum_{s=1}^{m} \phi_{s}=0$.

Similarly, results can be derived for $s=m+1, \ldots, n$, and $k=0, \ldots, m-1$.

In addition, a stationary cycle with all land allocated to forestry must satisfy $\lambda_{t} \geq 0$, for $t=0, \ldots$ Solving (12) or (13) for $\lambda_{t}$, eliminating $p_{s, t}, s=1, \ldots m-1$, $t=0, \ldots$, using $(20)$, we obtain

$$
\lambda_{t+k}=\frac{\left[U^{\prime}\left(c_{t+k}\right)+\beta p_{c} \theta\right] b^{m} f_{m}}{1-b^{m}}-\frac{\left[U^{\prime}\left(c_{t+1+k}\right)+\beta p_{c} \theta\right] b^{m+1} f_{m}}{1-b^{m}}-b W^{\prime}(0) \geq 0
$$

for $s=1, \ldots, m$, where $c_{t+1+m}=c_{t+1}$. Writing $c_{t+k}=f_{m} x_{s}$ and $c_{t+1+k}=$ $f_{m} x_{s-1}, s=1, \ldots, m$, where $x_{0}=x_{m}$ yields

$$
\lambda_{s}=\frac{\left[U^{\prime}\left(f_{m} x_{s}\right)+\beta p_{c} \theta\right] b^{m} f_{m}}{1-b^{m}}-\frac{\left[U^{\prime}\left(f_{m} x_{s-1}\right)+\beta p_{c} \theta\right] b^{m+1} f_{m}}{1-b^{m}}-b W^{\prime}(0) \geq 0
$$

for $s=1, \ldots, m$.

Given $g=\frac{\left[U^{\prime}\left(f_{m} / m\right)+\beta p_{c} \theta\right] b^{m} f_{m}}{1-b^{m}}-\frac{b}{1-b} W^{\prime}(0)>0$, there must exist a $\phi>0$ such that (25) is satisfied if $x_{s}=1 / m+\phi_{s}, s=1, \ldots, m$, for all $\left|\phi_{s}\right|<\phi$, $\sum_{s=1}^{m} \phi_{s}=0$. 
Let $i_{\infty}$ represent the stationary state level of variable $i$.

Corollary 2 If $g \equiv \frac{\left[U^{\prime}\left(f_{m} / m\right)+\beta p_{c} \theta\right] b^{m} f_{m}}{1-b^{m}}-\frac{b}{1-b} W^{\prime}(0) \leq 0$, optimal stationary cycles with $y_{\infty} \geq 0$ and $y_{\infty}$ constant do not exist.

Proof. Given $g \leq 0$, no solutions for (25) exist. Thus, by letting $\lambda_{t}=0$ in (12) or (13), eliminating $p_{s, t}, s=1, \ldots, m-1, t=0, \ldots$, using (20), and writing (12) analogously to (25), we obtain:

$$
\frac{\left[U^{\prime}\left(f_{m} x_{s}\right)+\beta p_{c} \theta\right] b^{m} f_{m}}{1-b^{m}}-\frac{\left[U^{\prime}\left(f_{m} x_{s-1}\right)+\beta p_{c} \theta\right] b^{m+1} f_{m}}{1-b^{m}}-b W^{\prime}\left(y_{\infty}\right) \geq 0
$$

for $s=1, \ldots, m$.

This system is linear in $\left[U^{\prime}\left(f_{m} x_{s}\right)+\beta p_{c} \theta\right], s=1, \ldots, m$. Its solution is given by

$$
U^{\prime}\left(f_{m} x_{s}\right)+\beta p_{c} \theta=\frac{W^{\prime}\left(y_{\infty}\right) \sum_{i=0}^{m-1} b^{i}}{b^{m-1} f_{m}}, s=1, \ldots, m
$$

as can be verified by direct substitution. Thus, $x_{s}=\left(1-y_{\infty}\right) / m, s=1, \ldots, m$ and optimal stationary cycles cannot exist.

Proposition 1 and Corollary 2 extend the results obtained in Salo and Tahvonen [3] to the case where carbon benefits are considered under a carbon flow regime. It is shown that, if carbon is fully released when harvest takes place, $(\theta=0)$, the carbon flow accounting regime will have no impact neither on the optimal forest management nor on the optimal land allocation between forest and other uses. This occurs because in this case the benefits of carbon sequestration are fully offset by the negative impact of carbon release when harvest takes place.

If , alternatively $(\theta>0)$, and if all land is forested land, the optimal forest management can lead to optimal cyclical harvesting, as shown in Proposition 1 , because smoothening an age class structure that deviates from normal forest is not optimal. From (23), we may conclude that when carbon sequestration 
benefits are accounted for, the cycle is larger than in the case without carbon sequestration benefits. Considering that the right hand side of (23) defines the maximized cycle radius, ${ }^{5}$ it is clear that for the same values of the parameters the term in brackets is greater than one. Therefore, the cycle radius is enlarged compared to the case without carbon benefits.

Finally, when it is optimal to allocate part of the forest land to alternative land use, the cycles that exist if all land is allocated to forestry are eliminated, and the remaining equilibrium is the normal forest steady state. However, again only if $(\theta>0)$, the optimal allocation area to forest will be increased. This can be derived from Corollary 2, but it is shown below in more detail.

\subsubsection{Stationary states}

Let assume that $g \leq 0$. Thus, we focus on interior solutions, that is, in the sense that land is used both in forestry and any alternative use. Corollary 2 above shows that in this case there cannot exist stationary cycles with constant $y_{\infty}$. We next show that in such cases there exists a stationary state that satisfies all the necessary conditions for optimality. ${ }^{6}$

Assuming that $m$ is unique, for a stationary state, we have that $p_{s, t}=p_{s, \infty}$, $c_{t}=c_{\infty}, y_{t}=y_{\infty}, \lambda_{t}=0$, and $x_{m, t}=x_{\infty}$, where $c_{\infty}, y_{\infty}, x_{\infty}$, and $p_{s, \infty}$, for $s=1, \ldots, n-1$, are constant. Direct substitution shows that

$$
p_{s}=W^{\prime}\left(y_{\infty}\right) \sum_{i=0}^{s-1} b^{-i}-f_{s}\left[U^{\prime}\left(C_{\infty}\right)+\beta p_{c} \theta\right], s=1, \ldots, n
$$

where $\sum_{i=0}^{s-1} b^{-i}=-b \frac{1-b^{-s}}{1-b}$

With some more algebra, we can write for $s=m$

$$
W^{\prime}\left(y_{\infty}\right) \frac{b}{1-b}-\frac{b^{m} f_{m}}{1-b^{m}} U^{\prime}\left(\frac{\left(1-y_{\infty}\right) f_{m}}{m}\right)-\frac{b^{m} f_{m}}{1-b^{m}} \beta p_{c} \theta=0
$$

\footnotetext{
${ }^{5}$ For a more detailed explanation see Salo and Tahvonen [1] pages 8-9 and 15 .

${ }^{6}$ The questions of convergence and stability of the stationary steady states are being studied.
} 
In this case, the allocation of land between forestry and the alternative use is optimal when the present value of output from a marginal unit of land in the alternative use equals the present value of a marginal use of bare forest land, where both timber value and the net benefits from carbon sequestration are accounted for. It is clear from (29) that, first if $(\theta=0)$, net carbon benefits in an optimal steady state are 0 . Second, if $(\theta>0)$ and assuming $m$ is unique, the first term has to be larger by concavity of $W$, and the second term has to be lower by concavity of $U$, implying that $y_{\infty}$ has to decrease. In fact, if trees also generate carbon benefits, given $m$, less land will be dedicated to the alternative use and more land will be put to forest. Moreover, at the steady-state, the incremental land area devoted to forest land will be evenly distributed among the different vintages.

\subsection{Tonne-year crediting}

The ton-year accounting method consists of crediting a forestry project with a fraction of its total yearly GHG benefit. This fraction is based on the stock of carbon stored each year, which is then converted, using $\left(E_{f}\right)$ to its equivalent amount of preventing effect. ${ }^{7}$

In this case, $S_{t}$ can be defined as follows:

$$
S_{t}=P_{c}\left(E_{f} \beta \sum_{s=1}^{n-1} f_{s} x_{s+1, t+1}\right)
$$

where the term in brackets represents the equivalent amount of emissions avoided in year $t$ due to the amount of carbon stored during year $t$. By considering $f_{s} x_{s+1, t+1}$, this formalization excludes from benefits' accounting all possible harvesting of younger age classes, in period $t$.

\footnotetext{
${ }^{7}$ Here, we consider $E_{f}$ constant. This assumption is consistent with Moura-Costa and Wilson' [11] approach, and also with Fearnside et al. [12], if in this last case we assume that the equivalence factor measures only the benefit of storing carbon in the forest for one additional year. To be fully consistent with Fearnside et al. [12], the equivalence factor should be different for each age class $s$, that is, $E_{f}(s)$.
} 
The decision problem in this case is similar to the previous one, except for the way carbon benefits are accounted for.

The necessary conditions for optimal solutions of problem (2-8) and $S_{t}$ given by (30), are similar to the previous case and are presented in Appendix 1 .

Based on this new formulation, and using a similar procedure to that used above, from the foc's we obtain a system of $m \times(m-1)$ equations:

$$
\begin{gathered}
b\left(p_{s+1, t+1+k}-p_{1, t+1+k}\right)-p_{s, t+k}=-b U^{\prime}\left(c_{t+1}\right)\left(f_{s+1}-f_{1}\right)+\left[U^{\prime}\left(c_{t}\right)-\beta p_{c} E_{f}\right] f_{s} \\
-b p_{1, t+1+k}-p_{m-1, t+k}=-b U^{\prime}\left(c_{t+1}\right)\left(f_{m}-f_{1}\right)+\left[U^{\prime}\left(c_{t}\right)-\beta p_{c} E_{f}\right] f_{m-1}
\end{gathered}
$$

where $s=1, \ldots m-2, k=0, \ldots, m-1$. This system is linear in the Lagragian multipliers $p_{s, t+k}, s=1, \ldots m-1, k=0, \ldots m-1$. Solving for any multiplier yields

$$
p_{s, t}=\frac{b^{m} f_{m}}{1-b^{m}}\left[b^{-s} U^{\prime}\left(c_{t+m-s}\right)-U^{\prime}\left(c_{t}\right)\right]-f_{s} U^{\prime}\left(c_{t}\right)+A_{s}
$$

where $A_{s}$ is given by

$$
A_{s}=\frac{\beta p_{c} E_{f}}{1-b^{m}}\left[\left(1-b^{m-s}\right)\left[\sum_{i=1}^{m-1}-b^{i} f_{i}\right]+\left(1-b^{m}\right) \sum_{j=s}^{m-1} b^{j-s} f_{j}\right]
$$

for $s=1, \ldots, m-1, t=0, \ldots$, as can be verified by direct substitution into the two equations above. In Appendix 2 we show that $A_{s}>0$, for $s=1, \ldots, m-1$.

\subsubsection{On the existence of stationary cycles}

Proposition 3 Given $g \equiv \frac{U^{\prime}\left(f_{m} / m\right) b^{m} f_{m}}{1-b^{m}}+\frac{\beta p_{c} E_{f}}{1-b^{m}}\left(\sum_{i=1}^{m-1} b^{i} f_{i}\right)-\frac{b}{1-b} W^{\prime}(0)>0$, $m \geq 2$, and $b<1$, there exists a set of interior Optimal Faustmann Forests with $\phi^{k}>0$. 
Proof. Following Salo and Tahvonen [3], condition (61) requires, for the indefinitely repeated cycle, that $p_{s, t+k} \geq 0$ for $s=1, \ldots m-1, k=0, \ldots, m-1$. Thus, the fact that $x \in K$ implies by (33) that

$$
\frac{U^{\prime}\left(c_{t+k}\right)}{U^{\prime}\left(c_{t+k+m-j}\right)} \leq \frac{b^{m-j} f_{m}}{f_{j}+b^{m}\left(f_{m}-f_{j}\right)}+\frac{A_{j}\left(1-b^{m}\right)}{\left[f_{j}+b^{m}\left(f_{m}-f_{j}\right)\right] U^{\prime}\left(c_{t+k+m-j}\right)}
$$

for $k=0, \ldots, m-1, j=1, \ldots m-1$, where $A_{j}$ is given by (34). Using (3) and the definition of Faustmann harvesting, we can write $c_{t+k}=f_{m} x_{s}$ and $c_{t+k+m-j}=f_{m} x_{s-m+j}$ where $s-m+j$ is understood as $s-j$, if $s-m+j \leq 0$. Equation (35) takes the form

$$
\frac{U^{\prime}\left(f_{m} x_{s}\right)}{U^{\prime}\left(f_{m} x_{s-1}\right)} \leq \frac{b^{m-j} f_{m}}{f_{j}+b^{m}\left(f_{m}-f_{j}\right)}+\frac{A_{j}\left(1-b^{m}\right)}{\left[f_{j}+b^{m}\left(f_{m}-f_{j}\right)\right] U^{\prime}\left(f_{m} x_{s-1}\right)}
$$

or, alternatively,

$$
\frac{U^{\prime}\left(f_{m} x_{s}\right)}{U^{\prime}\left(f_{m} x_{s-1}\right)} \leq \eta_{j}\left[1+\frac{A_{j}\left(1-b^{m}\right)}{U^{\prime}\left(f_{m} x_{s-1}\right) b^{m-j} f_{m}}\right]
$$

for $s=1, \ldots m, j=1, \ldots, m-1$. Since $\eta_{j} \equiv \frac{b^{m-j} f_{m}}{f_{j}+b^{m}\left(f_{m}-f_{j}\right)}>1, j=1, \ldots, m-1$, if $A_{s} \geq 0$ the second term is always larger than one. Then, by the strict concavity of $U$, and since the right-hand side of (36) is larger than 1 , there must exist a $\phi>0$, such that (36) is satisfied if $x_{s}=1 / m+\phi_{s}, s=1, \ldots, m$, for all $\left|\phi_{s}\right|<\phi$, $\sum_{s=1}^{m} \phi_{s}=0$.

Similarly, results can be derived for $s=m+1, \ldots, n$, and $k=0, \ldots, m-1$.

In addition, a stationary cycle with all land allocated to forestry must satisfy $\lambda_{t} \geq 0$, for $t=0, \ldots$ Solving (57) or (58) for $\lambda_{t}$, eliminating $p_{s, t}, s=1, \ldots m-1$, $t=0, \ldots$, using (33), we obtain

$$
\lambda_{t+k}=\frac{U^{\prime}\left(c_{t+k}\right) b^{m} f_{m}}{1-b^{m}}-\frac{U^{\prime}\left(c_{t+1+k}\right) b^{m+1} f_{m}}{1-b^{m}}+\frac{\beta p_{c} E_{f}}{1-b^{m}}\left(\sum_{i=1}^{m-1} b^{i}(1-b) f_{i}\right)-b W^{\prime}(0) \geq 0
$$

for $s=1, \ldots, m$, where $c_{t+1+m}=c_{t+1}$. Writing $c_{t+k}=f_{m} x_{s}$ and $c_{t+1+k}=$ 
$f_{m} x_{s-1}, s=1, \ldots, m$, where $x_{0}=x_{m}$ yields

$\lambda_{s}=\frac{U^{\prime}\left(f_{m} x_{s}\right) b^{m} f_{m}}{1-b^{m}}-\frac{U^{\prime}\left(f_{m} x_{s-1}\right) b^{m+1} f_{m}}{1-b^{m}}+\frac{\beta p_{c} E_{f}}{1-b^{m}}\left(\sum_{i=1}^{m-1} b^{i}(1-b) f_{i}\right)-b W^{\prime}(0) \geq 0$

for $s=1, \ldots, m$.

Given $g=\frac{U^{\prime}\left(f_{m} / m\right) b^{m} f_{m}}{1-b^{m}}+\frac{\beta p_{c} E_{f}}{1-b^{m}}\left(\sum_{i=1}^{m-1} b^{i} f_{i}\right)-\frac{b}{1-b} b W^{\prime}(0)>0$, there must exist a $\phi>0$ such that (39) is satisfied if $x_{s}=1 / m+\phi_{s}, s=1, \ldots, m$, for all $\left|\phi_{s}\right|<\phi, \sum_{s=1}^{m} \phi_{s}=0$.

Again, let $i_{\infty}$ represent the stationary state level of variable $i$.

Corollary 4 If $g \equiv \frac{U^{\prime}\left(f_{m} / m\right) b^{m} f_{m}}{1-b^{m}}+\frac{\beta p_{c} E_{f}}{1-b^{m}}\left(\sum_{i=1}^{m-1} b^{i} f_{i}\right)-\frac{b}{1-b} W^{\prime}(0) \leq 0$, optimal stationary cycles with $y_{\infty} \geq 0$ and $y_{\infty}$ constant do not exist.

Proof. Given $g \leq 0$, no solutions for (39) exist. Thus, by letting $\lambda_{t}=0$ in (57) or (58), eliminating $p_{s, t}, s=1, \ldots, m-1, t=0, \ldots$, using (33), and writing (57) analogously to (39), we obtain:

$\frac{U^{\prime}\left(f_{m} x_{s}\right) b^{m} f_{m}}{1-b^{m}}-\frac{U^{\prime}\left(f_{m} x_{s-1}\right) b^{m+1} f_{m}}{1-b^{m}}+\frac{\beta p_{c} E_{f}}{1-b^{m}}\left(\sum_{i=1}^{m-1} b^{i}(1-b) f_{i}\right)-b W^{\prime}\left(y_{\infty}\right) \geq 0$

for $s=1, \ldots, m$.

This system is linear in $U^{\prime}\left(f_{m} x_{s}\right), s=1, \ldots, m$. Its solution is given by

$$
U^{\prime}\left(f_{m} x_{s}\right)+\frac{p_{c} \beta E_{f}\left(\sum_{i=1}^{m-1} b^{i} f_{i}\right)}{b^{m} f_{m}}=\frac{W^{\prime}\left(y_{\infty}\right) \sum_{i=0}^{m-1} b^{i}}{b^{m-1} f_{m}}, s=1, \ldots, m
$$

as can be verified by direct substitution. Thus, $x_{s}=\left(1-y_{\infty}\right) / m, s=1, \ldots, m$ and optimal stationary cycles cannot exist.

Proposition 3 and Corollary 4 extend the results obtained in Salo and Tahvonen [3] for the case where carbon benefits are considered under a tonne year credit regime. In this case, both the optimal land allocation and the optimal forest management are changed when introducing carbon sequestration benefits. 
Again, if all land is forested land, optimal forest management can lead to optimal cyclical harvesting. Moreover, the cycles increase when compared to the case without carbon sequestration benefits, as $A_{s}>0$ for $s=1, \ldots, m-1$, implying that the right-hand side of (37) is larger than $\eta_{j}$.

When it is optimal to allocate part of the forest land to alternative land use, from Corollary 4 we conclude that the cycles are eliminated, and the remaining equilibrium is again the normal forest steady state. In this case, the optimal allocation area to forest will always increase, due to the fact that carbon sequestration benefits have always a positive net value. The stationary state is developed below.

\subsubsection{Stationary states}

Let assume again that $g \leq 0$ and focus on interior solutions, that is, in the sense that land is used both in forestry and any alternative use. Corollary 4 above shows that in this case there cannot exist stationary cycles with constant $y_{\infty}$. We next show that in such cases there exists a stationary state that satisfies all the necessary conditions for optimality.

Assuming again that $m$ is unique, for a stationary state, we have that $p_{s, t}=$ $p_{s, \infty}, c_{t}=c_{\infty}, y_{t}=y_{\infty}, \lambda_{t}=0$, and $x_{m, t}=x_{\infty}$, where $c_{\infty}, y_{, \infty}, x_{\infty}$, and $p_{s, \infty}$, for $s=1, \ldots, n-1$, are constant. Direct substitution shows that

$$
p_{s}=W^{\prime}\left(y_{\infty}\right) \sum_{j=0}^{s-1} b^{-j}-f_{s} U^{\prime}\left(C_{\infty}\right)-b^{-s} \beta p_{c} E_{f}\left(\sum_{i=1}^{m-1} b^{i} f_{i}-\sum_{i=s}^{m-1} b^{i} f_{i}\right)
$$

for $s=1, \ldots, n$, where $\sum_{j=0}^{s-1} b^{-j}=-b \frac{1-b^{-s}}{1-b}$,

With some more algebra, we can write for $s=m$

$$
W^{\prime}\left(y_{\infty}\right) \frac{b}{1-b}-\frac{b^{m} f_{m}}{1-b^{m}} U^{\prime}\left(\frac{\left(1-y_{\infty}\right) f_{m}}{m}\right)-\frac{\beta p_{c} E_{f}}{1-b^{m}} \sum_{i=1}^{m-1} b^{i} f_{i}=0
$$

Therefore, the allocation of land between forestry and the alternative use is optimal when the present value of output from a marginal unit of land in the 
alternative use equals the present value of a marginal use of bare forest land. In this case, the net benefits from carbon sequestration (third term of (43)) are the present value of "emissions equivalence reduction" of a marginal unit of forest bare land with a rotation period of dimension $m$.

Since $m$ is unique, it is clear from (43) that the first term has to be larger and the second term has to be lower by concavity of $W$, and $U$, implying that $y_{\infty}$ has to decrease. In fact, if trees also generate carbon benefits, given $m$, less land will be dedicated to the alternative use and more land will be put to forest. Moreover, at the steady-state, the incremental forest land area will be evenly distributed among the different vintages.

\subsection{Average Storage Method}

The average storage accounting method consists of crediting a forestry project with the amount of yearly carbon benefits that the land allocated to forest generates, on average, at the end of each rotation. This average is calculated using a constant weight, which corresponds to the average amount of the carbon stock stored, which is applied to every class $s$ area of forest land, for $s=$ $1, \ldots, m-1$. In this case, and without loss of generality, we apply the average to the next period class $s$ land allocation, as it makes easier the comparison with the ton-year crediting case.

In this case, $S_{t}$ can be defined as follows:

$$
S_{t}=P_{c} \beta \frac{\sum_{s=1}^{m-1} f_{s}}{m} \sum_{s=1}^{n-1} x_{s+1, t+1}
$$

Again, the decision problem is similar to the previous one, except for the way carbon benefits are accounted for. The necessary conditions for optimal solutions of problem (2-8) and $S_{t}$ given by (44), are similar to the previous case and are presented in Appendix 3.

Based on this new formulation, and using a similar procedure to that used 
above, from the foc's we obtain a system of $m \times(m-1)$ equations:

$$
\begin{gathered}
b\left(p_{s+1, t+1+k}-p_{1, t+1+k}\right)-p_{s, t+k}=-b U^{\prime}\left(c_{t+1}\right)\left(f_{s+1}-f_{1}\right)+f_{s} U^{\prime}\left(c_{t}\right)-b D \\
-b p_{1, t+1+k}-p_{m-1, t+k}=-b U^{\prime}\left(c_{t+1}\right)\left(f_{m}-f_{1}\right)+f_{m-1} U^{\prime}\left(c_{t}\right)-b D
\end{gathered}
$$

where $s=1, \ldots m-2, k=0, \ldots, m-1$, and where $D=P_{c} \beta \frac{\sum_{s=1}^{m-1} f_{s}}{m}$.

This system is linear in the Lagragian multipliers $p_{s, t+k}, s=1, \ldots m-1$, $k=0, \ldots m-1$. Solving for any multiplier yields

$$
p_{s, t}=\frac{b^{m} f_{m}}{1-b^{m}}\left[b^{-s} U^{\prime}\left(c_{t+m-s}\right)-U^{\prime}\left(c_{t}\right)\right]-f_{s} U^{\prime}\left(c_{t}\right)+B_{s}
$$

where $B_{s}$ is given by

$$
B_{s}=\frac{b\left(1-b^{m-s}\right)}{1-b^{m}} D
$$

for $s=1, \ldots, m-1, t=0, \ldots$, as can be verified by direct substitution into the two equations above. By inspection, it is clear that $B_{s}>0$, for $s=1, \ldots, m-1$, $t=0, \ldots \ldots$

\subsubsection{On the existence of stationary cycles}

Proposition 5 Given $g \equiv \frac{U^{\prime}\left(f_{m} / m\right) b^{m} f_{m}}{1-b^{m}}+\frac{b}{1-b} D-\frac{b}{1-b} W^{\prime}(0)>0, m \geq 2$, and $b<1$, there exists a set of interior Optimal Faustmann Forests with $\phi^{k}>0$.

Proof. Following Salo and Tahvonen [3], condition (72) requires, for the indefinitely repeated cycle, that $p_{s, t+k} \geq 0$ for $s=1, \ldots m-1, k=0, \ldots, m-1$. Thus, the fact that $x \in K$ implies by (47) that

$$
\frac{U^{\prime}\left(c_{t+k}\right)}{U^{\prime}\left(c_{t+k+m-j}\right)} \leq \frac{b^{m-j} f_{m}}{f_{j}+b^{m}\left(f_{m}-f_{j}\right)}+\frac{B_{j}\left(1-b^{m}\right)}{\left[f_{j}+b^{m}\left(f_{m}-f_{j}\right)\right] U^{\prime}\left(c_{t+k+m-j}\right)}
$$

for $k=0, \ldots, m-1, j=1, \ldots m-1$, where $B_{j}$ is given by (48). Using (3) and the definition of Faustmann harvesting, we can write $c_{t+k}=f_{m} x_{s}$ and 
$c_{t+k+m-j}=f_{m} x_{s-m+j}$ where $s-m+j$ is understood as $s-j$, if $s-m+j \leq 0$.

Equation (49) takes the form

$$
\frac{U^{\prime}\left(f_{m} x_{s}\right)}{U^{\prime}\left(f_{m} x_{s-1}\right)} \leq \frac{b^{m-j} f_{m}}{f_{j}+b^{m}\left(f_{m}-f_{j}\right)}+\frac{B_{j}\left(1-b^{m}\right)}{\left[f_{j}+b^{m}\left(f_{m}-f_{j}\right)\right] U^{\prime}\left(f_{m} x_{s-1}\right)}
$$

for $s=1, \ldots m, j=1, \ldots, m-1$. Since $\eta_{j} \equiv \frac{b^{m-j} f_{m}}{f_{j}+b^{m}\left(f_{m}-f_{j}\right)}>1, j=1, \ldots, m-1$, and $B_{s}>0$, the second term is always positive. Then, by the strict concavity of $U$, and since the right-hand side of (50) is larger than 1 , there must exist a $\phi>0$, such that (50) is satisfied if $x_{s}=1 / m+\phi_{s}, s=1, \ldots, m$, for all $\left|\phi_{s}\right|<\phi$, $\sum_{s=1}^{m} \phi_{s}=0$.

Similarly, results can be derived for $s=m+1, \ldots, n$, and $k=0, \ldots, m-1$.

In addition, a stationary cycle with all land allocated to forestry must satisfy $\lambda_{t} \geq 0$, for $t=0, \ldots$ Solving (68) or (69) for $\lambda_{t}$, eliminating $p_{s, t}, s=1, \ldots m-1$, $t=0, \ldots$, using $(47)$, we obtain

$$
\lambda_{t+k}=\frac{U^{\prime}\left(c_{t+k}\right) b^{m} f_{m}}{1-b^{m}}-\frac{U^{\prime}\left(c_{t+1+k}\right) b^{m+1} f_{m}}{1-b^{m}}+b D-b W^{\prime}(0) \geq 0
$$

for $s=1, \ldots, m$, where $c_{t+1+m}=c_{t+1}$. Writing $c_{t+k}=f_{m} x_{s}$ and $c_{t+1+k}=$ $f_{m} x_{s-1}, s=1, \ldots, m$, where $x_{0}=x_{m}$ yields

$$
\lambda_{s}=\frac{U^{\prime}\left(f_{m} x_{s}\right) b^{m} f_{m}}{1-b^{m}}-\frac{U^{\prime}\left(f_{m} x_{s-1}\right) b^{m+1} f_{m}}{1-b^{m}}+b D-b W^{\prime}(0) \geq 0
$$

for $s=1, \ldots, m$.

Given $g=\frac{U^{\prime}\left(f_{m} / m\right) b^{m} f_{m}}{1-b^{m}}+\frac{b}{1-b} D-\frac{b}{1-b} b W^{\prime}(0)>0$, there must exist a $\phi>0$ such that (52) is satisfied if $x_{s}=1 / m+\phi_{s}, s=1, \ldots, m$, for all $\left|\phi_{s}\right|<\phi$, $\sum_{s=1}^{m} \phi_{s}=0$.

Once more, let $i_{\infty}$ represent the stationary state level of variable $i$.

Corollary 6 If $g \equiv \frac{U^{\prime}\left(f_{m} / m\right) b^{m} f_{m}}{1-b^{m}}+\frac{b}{1-b} D-\frac{b}{1-b} W^{\prime}(0) \leq 0$, optimal stationary cycles with $y_{\infty} \geq 0$ and $y_{\infty}$ constant do not exist.

Proof. Given $g \leq 0$, no solutions for (52) exist. Thus, by letting $\lambda_{t}=0$ in (68) or (69), eliminating $p_{s, t}, s=1, \ldots, m-1, t=0, \ldots$, using (47), and writing 
(68) analogously to (52), we obtain:

$$
\frac{U^{\prime}\left(f_{m} x_{s}\right) b^{m} f_{m}}{1-b^{m}}-\frac{U^{\prime}\left(f_{m} x_{s-1}\right) b^{m+1} f_{m}}{1-b^{m}}+b D-b W^{\prime}\left(y_{\infty}\right) \geq 0
$$

for $s=1, \ldots, m$.

This system is linear in $U^{\prime}\left(f_{m} x_{s}\right), s=1, \ldots, m$. Its solution is given by

$$
U^{\prime}\left(f_{m} x_{s}\right)+\frac{\sum_{i=0}^{m-1} b^{i}}{b^{m-1} f_{m}} D=\frac{W^{\prime}\left(y_{\infty}\right) \sum_{i=0}^{m-1} b^{i}}{b^{m-1} f_{m}}, s=1, \ldots, m
$$

as can be verified by direct substitution. Thus, $x_{s}=\left(1-y_{\infty}\right) / m, s=1, \ldots, m$ and optimal stationary cycles cannot exist.

Proposition 5 and Corollary 6 extend the results obtained in Salo and Tahvonen [3] to the case where carbon benefits are calculated based on the average stock regime. Under this accounting method, considering carbon benefits also impacts both the optimal land allocation and the optimal forest management.

Again, if all land is forested land, optimal forest management can lead to optimal cyclical harvesting and from (50), as $B_{s}>0$, it is clear that the average carbon storage accounting method also increases the cycles dimension.

When it is optimal to allocate part of the forest land to alternative land use, the cycles are eliminated, and the remaining equilibrium is once more the normal forest steady state. The optimal allocation area to forest will again always increase, due to the fact that this carbon benefits accounting method has a positive net value.

\subsubsection{Stationary states}

Let assume again that $g \leq 0$ and focus on interior solutions, that is, in the sense that land is used both in forestry and any alternative use. Corollary 6 above shows that in this case there cannot exist stationary cycles with constant $y_{\infty}$. We next show that in such cases there exists a stationary state that satisfies all the necessary conditions for optimality. 
Assuming again that $m$ is unique, for a stationary state, we have that $p_{s, t}=$ $p_{s, \infty}, c_{t}=c_{\infty}, y_{t}=y_{\infty}, \lambda_{t}=0$, and $x_{m, t}=x_{\infty}$, where $c_{\infty}, y_{\infty}, x_{\infty}$, and $p_{s, \infty}$, for $s=1, \ldots, n-1$, are constant. Direct substitution shows that

$$
p_{s}=W^{\prime}\left(y_{\infty}\right) \sum_{j=0}^{s-1} b^{-j}-f_{s} U^{\prime}\left(C_{\infty}\right)-\sum_{j=0}^{s-1} b^{-j} D
$$

for $s=1, \ldots, n$, where $\sum_{j=0}^{s-1} b^{-j}=-b \frac{1-b^{-s}}{1-b}$,

With some more algebra, we can write for $s=m$

$$
W^{\prime}\left(y_{\infty}\right) \frac{b}{1-b}-\frac{b^{m} f_{m}}{1-b^{m}} U^{\prime}\left(\frac{\left(1-y_{\infty}\right) f_{m}}{m}\right)-\frac{b}{1-b} D=0
$$

Therefore, the allocation of land between forestry and the alternative use is optimal when the present value of output from a marginal unit of land in the alternative use equals the present value of a marginal use of bare forest land. Here, the net benefits from carbon sequestration (third term of (56)) are the present value of the yearly constant payment to a marginal unit of forest land, $D$.

Since $m$ is unique, it is clear from (56) that $y_{\infty}$ has to decrease. Also, more land will be put to forest, and, at the steady-state, the incremental forest land area will be evenly distributed among the different vintages.

\section{Conclusion}

The introduction of carbon sequestration benefits in the multiple vintage forest model developed by Salo and Tahvonen [3] is undertaken by considering different carbon accounting methods, namely, the carbon flow regime, the ton-year crediting and the average carbon storage. In general, we conclude that the main results obtained in Salo and Tahvonen still apply. In fact, if all land is forested land, optimal forest management can lead to optimal cyclical harvesting because smoothening an age class structure that deviates from normal forest is not optimal. When it is optimal to allocate part of the forest land to alternative 
land use, the cycles that exist if all land is allocated to forestry are eliminated, and the remaining equilibrium is the normal forest steady state.

Although the major theoretical results still apply, the extension to the presence of carbon sequestration benefits is not without consequences. In fact, we conclude that different accounting methods for carbon sequestration benefits determine different impacts and their dimension depends largely on the empirical parameters values.

First, in the case that all land is forested land, under the optimal cyclical harvesting, the cycles are increased when compared to the case without carbon benefits. Second, the optimal allocation area to forest will, in general, increase, as the net value from accounting carbon sequestration benefits is positive.

Formally, it is not possible to compare the impact dimension of the different accounting methods both on the cycles dimension and on the optimal land allocation because they are based on distinct concepts, $\theta, E_{f}$, and $D$, respectively. However, empirically, depending on the values taken by the different parameters, comparisons may eventually be undertaken. Thus, aside from numerical simulations, some insights can be found. For example, if the value of $\theta$ is very small, close to that of $E_{f}$, we observe that the impact in the tonne-year crediting case is greater than in the carbon flow one.

When the carbon flow accounting method is considered, the impact on optimal forest management will depend upon the amount of carbon released when the forest is harvested. In fact, if all the carbon is released at harvest $(\theta=0)$, carbon sequestration benefits will have no impact on the optimal management and allocation of land. The cumulative benefits from forest use over time are zero. In addition, in this case, the carbon price level is irrelevant as an incentive to forest carbon sequestration.

In the limit case of $(\theta=1)$, carbon sequestration benefits will have the maximum possible impact on the optimal allocation of land, increasing the 
forest land area. Here, the cumulative benefits from forest use over time are positive due to the fact that some carbon is "permanently" stored in long run structures.

When the tonne year crediting or the average storage accounting system are considered, the optimal forest management changes. The optimal allocation of land between forest and other uses increases the forested area. This is due to the fact that in these cases the benefits from forest use are always positive.

To conclude, the theoretical model developed in this paper can be a useful tool to empirical studies on forestry policy in general, or, in particular, to examine the impact of policy measures to reduce GHG emissions or to implement the Kyoto Protocol. 


\section{Appendix 1}

The Karush-Kuhn-Tucker conditions for optimal solutions of problem (2-8) and $S_{t}$ given by (30) for all $t=0, \ldots$, are given by:

$$
\begin{gathered}
b^{t} \frac{\partial L}{\partial x_{1, t+1}}=b f_{1} U^{\prime}\left(c_{t+1}\right)-b W^{\prime}\left(y_{t+1}\right)-\lambda_{t}+b p_{1, t+1} \leq 0 \\
b^{t} \frac{\partial L}{\partial x_{s+1, t+1}}=-f_{s} U^{\prime}\left(c_{t}\right)+b f_{s+1} U^{\prime}\left(c_{t+1}\right)+f_{s} p_{c} \beta E_{f}- \\
-b W^{\prime}\left(y_{t+1}\right)-\lambda_{t}+b p_{s+1, t+1}-p_{s, t} \leq 0
\end{gathered}
$$

for $s=1, \ldots, n-2$,

$b^{t} \frac{\partial L}{\partial x_{n, t+1}}=-f_{n-1} U^{\prime}\left(c_{t}\right)+b f_{n} U^{\prime}\left(c_{t+1}\right)+f_{n-1} p_{c} \beta E_{f}-b W^{\prime}\left(y_{t+1}\right)-\lambda_{t}-p_{n-1, t} \leq 0$

$$
\begin{gathered}
x_{s, t+1} \geq 0, x_{s, t+1} \frac{\partial L}{\partial x_{s, t+1}}=0, s=1, \ldots, n \\
p_{s, t} \geq 0, p_{s, t}\left(x_{s, t}-x_{s+1, t+1}\right)=0, s=1, \ldots, n-1 \\
\lambda_{t} \geq 0, \lambda_{t}\left(1-\sum_{s=1}^{n} x_{s, t+1}\right)=0
\end{gathered}
$$

The existence of optimal solutions for bounded utility and $b<1$ follows from Theorem 4.6 in Stokey and Lucas (p.79).

\section{Appendix 2}

Lemma $7 A_{s}>0$, for $s=1, \ldots, m-1$. 
Proof. From (34), and with some algebra $A_{s}>0$ implies that:

$$
\sum_{i=0}^{m-1} b^{i} \sum_{j=s}^{m-1} b^{j-s} f_{j}>\sum_{j=0}^{m-s-1} b^{j} \sum_{i=1}^{m-1} b^{i} f_{i}
$$

which can be rewritten as:

$$
\begin{aligned}
& \sum_{j=s}^{m-1} b^{j-s} f_{j}+\sum_{j=s}^{m-1} b^{j+1-s} f_{j}+\ldots+\sum_{j=s}^{m-1} b^{j-1} f_{j}+\sum_{j=s}^{m-1} b^{j} f_{j}+\sum_{j=s}^{m-1} b^{j+1} f_{j}+\ldots+\sum_{j=s}^{m-1} b^{j+m-1-s} f_{j}> \\
& \sum_{i=1}^{s-1} b^{i} f_{i}+\sum_{i=s}^{m-1} b^{i} f_{i}+\sum_{i=1}^{s-1} b^{i+1} f_{i}+\sum_{i=s}^{m-1} b^{i+1} f_{i}+\ldots+\sum_{i=1}^{s-1} b^{i+m-s-1} f_{i}+\sum_{i=s}^{m-1} b^{i+m-s-1} f_{i}
\end{aligned}
$$

Eliminating the equal terms in both sides of the inequality and rearranging the remaining sums of (64), we have:

$$
\begin{aligned}
\sum_{j=s}^{m-1} b^{j-s} f_{j}+ & \sum_{i=1}^{s-1} b^{i} f_{s}+\sum_{i=1}^{s-1} b^{i+1} f_{s+1}+\ldots .+\sum_{i=1}^{s-1} b^{i+m-s-1} f_{m-1}> \\
& \sum_{i=1}^{s-1} b^{i} f_{i}+\sum_{i=1}^{s-1} b^{i+1} f_{i}+\ldots .+\sum_{i=1}^{s-1} b^{i+m-s-1} f_{i}
\end{aligned}
$$

Since $\sum_{j=s}^{m-1} b^{j-s} f_{j}>0$, for $b<1$, and $\left(\sum_{j=s}^{m-1} \sum_{i=1}^{s-1} b^{i}\left(f_{j}-f_{i}\right)>0\right.$, the result follows. Moreover, from (34), we observe that $A_{m}=0$, and that $A_{s}$ decreases to zero as $s$ increases to $m$.

\section{Appendix 3}

The Karush-Kuhn-Tucker conditions for optimal solutions of problem (2-8) and $S_{t}$ given by (44) for all $t=0, \ldots$, are given by:

$$
b^{t} \frac{\partial L}{\partial x_{1, t+1}}=b f_{1} U^{\prime}\left(c_{t+1}\right)-b W^{\prime}\left(y_{t+1}\right)-\lambda_{t}+b p_{1, t+1} \leq 0
$$




$$
\begin{gathered}
b^{t} \frac{\partial L}{\partial x_{s+1, t+1}}=-f_{s} U^{\prime}\left(c_{t}\right)+b f_{s+1} U^{\prime}\left(c_{t+1}\right)+b D- \\
-b W^{\prime}\left(y_{t+1}\right)-\lambda_{t}+b p_{s+1, t+1}-p_{s, t} \leq 0
\end{gathered}
$$

for $s=1, \ldots, n-2$,

$b^{t} \frac{\partial L}{\partial x_{n, t+1}}=-f_{n-1} U^{\prime}\left(c_{t}\right)+b f_{n} U^{\prime}\left(c_{t+1}\right)+b D-b W^{\prime}\left(y_{t+1}\right)-\lambda_{t}-p_{n-1, t} \leq 0$

$$
\begin{gathered}
x_{s, t+1} \geq 0, x_{s, t+1} \frac{\partial L}{\partial x_{s, t+1}}=0, s=1, \ldots, n \\
p_{s, t} \geq 0, p_{s, t}\left(x_{s, t}-x_{s+1, t+1}\right)=0, s=1, \ldots, n-1 \\
\lambda_{t} \geq 0, \lambda_{t}\left(1-\sum_{s=1}^{n} x_{s, t+1}\right)=0
\end{gathered}
$$

where $D=P_{c} \beta \frac{\sum_{s=1}^{m-1} f_{s}}{m}$. The existence of optimal solutions for bounded utility and $b<1$ follows from Theorem 4.6 in Stokey and Lucas (p.79). 


\section{References}

[1] Salo, S. and O. Tahvonen, 2002, "On Equilibrium Cycles and Normal Forests in Optimal Harvesting of Tree Vintages", Journal of Environmental Economics and Management, 44, 1-22.

[2] Salo, S. and O. Tahvonen, 2003, "On the Economics of Forest Vintages", Journal Economics Dynamics and Control, 27, 1411-1435.

[3] Salo, S. and O. Tahvonen, 2004, "Renewable Resources with Endogenous Age Classes and Allocation of Land", Amer. J. Agri. Economics, 86(2), 513-530.

[4] Sohngen, B. and R. Mendelsohn, 2003, "An Optimal Control Model of Forest Carbon Sequestration", Amer. J. Agric. Econ. 85, 2, 448-457.

[5] Van Kooten, G. C., C. S. Binkley and G. Delcourt, 1995, "Effect of Carbon Taxes and Subsidies on Optimal Forest Rotation Age and Supply of Carbon Services", American Journal of Agricultural Economics, vol. 77, 2 , 365-374.

[6] Cunha-e-Sá, M. and R. Rosa, 2006, "Impact of Carbon Accounting Methods on Optimal Forest Management: An Application to the Portuguese Eucalyptus Forest", mimeo.

[7] Velt, K. van't,and A. Plantinga, 2005, "Carbon Sequestration or Abatement? The Effect of Rising Carbon Prices on the Optimal Portfolio of Greenhouse-Gas Mitigation Strategies", Journal of Environmental Economics and Management.

[8] Spring, D, J. Kennedy, J. and R. Nally, 2005, "Optimal Management of a forested catchment providing timber and carbon sequestration benefits: Climate Change Effects", Global Environmental Change, 15, 281-292. 
[9] IPCC (Intergovernmental Panel on Climate Change), 2000. Land Use, Land-Use Change, and Forestry. Special Report of the Intergovernmental Panel on Climate Change. Robert T. Watson, Ian R. Noble, Bert Bolin, N. H. Ravindranath, David J. Verardo and David J.Dokken (Eds.). Cambridge University Press, UK.

[10] Locatelli, B. and L. Pedroni, 2003, "Rewarding carbon sequestration in forest plantation projects: how the CDM could be attractive to small stakeholders? "Working Paper, Global Change Group, CATIE, Turrialba, Costa Rica.

[11] Moura-Costa, P.H. and C. Wilson, 2000, "An equivalence factor between $\mathrm{CO} 2$ avoided emissions and sequestration-Description and applications in forestry," Mitigation and Adaptation Strategies for Global Change, 5:51-60.

[12] Fearnside P. M., D. A. Lashof and P. H. Moura-Costa, 2000, "Accounting for time in mitigating global warming, Mitigation and Adaptation Strategies for Global Change 5(3): 230-270. Available at: http://www.kluweronline.com/issn/1381-2386. 